\title{
PABELLÓN DE AULAS PARA LA FACULTAD DE CIENCIAS EN EL CAMPUS DE LA UNIVERSIDAD DE ALCALA DE HENARES/MADRID/ESPAÑA
}

\section{(CLASSROOM BUILDOIG FOR THE FACULTY OF SCIENCES IN THE CAMPUS OF THE UNI- VERSITY OF ALCALÁ DE HENARES/MADRID/SPAIN)}

Carmelo Oñate Gómez, Dr. Arquitecto

\section{RESUMEN}

Presentamos un edificio destinado a Pabellón de aulas en el que se ha pretendido conseguir una solución funcional, contenedora, diferenciando claramente los espacios comunes y de circulación con vocación integradora en el paisaje, y las aulas entendidas como un espacio docente participativo.

La forma debe ayudar a la función y especialmente en las aulas se necesita crear un ambiente direccional que colabore al coloquio.

El acondicionamiento acústico, para conseguir la mejor inteligibilidad de la palabra, exige utilizar soluciones constructivas adecuadas que permitan mantener un nivel de inmisión propio de una habitación media-muerta, con un alto nivel de ruido en el exterior.

La utilización de materiales de acabado debe conseguir ajustar la curva frecuencia-tiempo de reverberación al tiempo óptimo, sin necesidad de utilizar medios electroacústicos.

\section{SUMMARY}

We present here a building designed for classrooms. The designer's intention was to create a functional, comprehensive facility where common areas and corridors, integrated in the landscape, were clearly differenciated from the classrooms conceived as a participative learning space.

Form is to help function and, particularly, a directional environment is to be created in classrooms to promote discussion.

Acoustic conditioning requieres, to achieve the best possible understanding of speech, using appropriate construction techniques to create an inmission level typical of a dead medium room with a high level of external noise.

Finishing materials are used to adjust the frequency. reverberation time to the optimal period without recourse to electro-acoustic means.

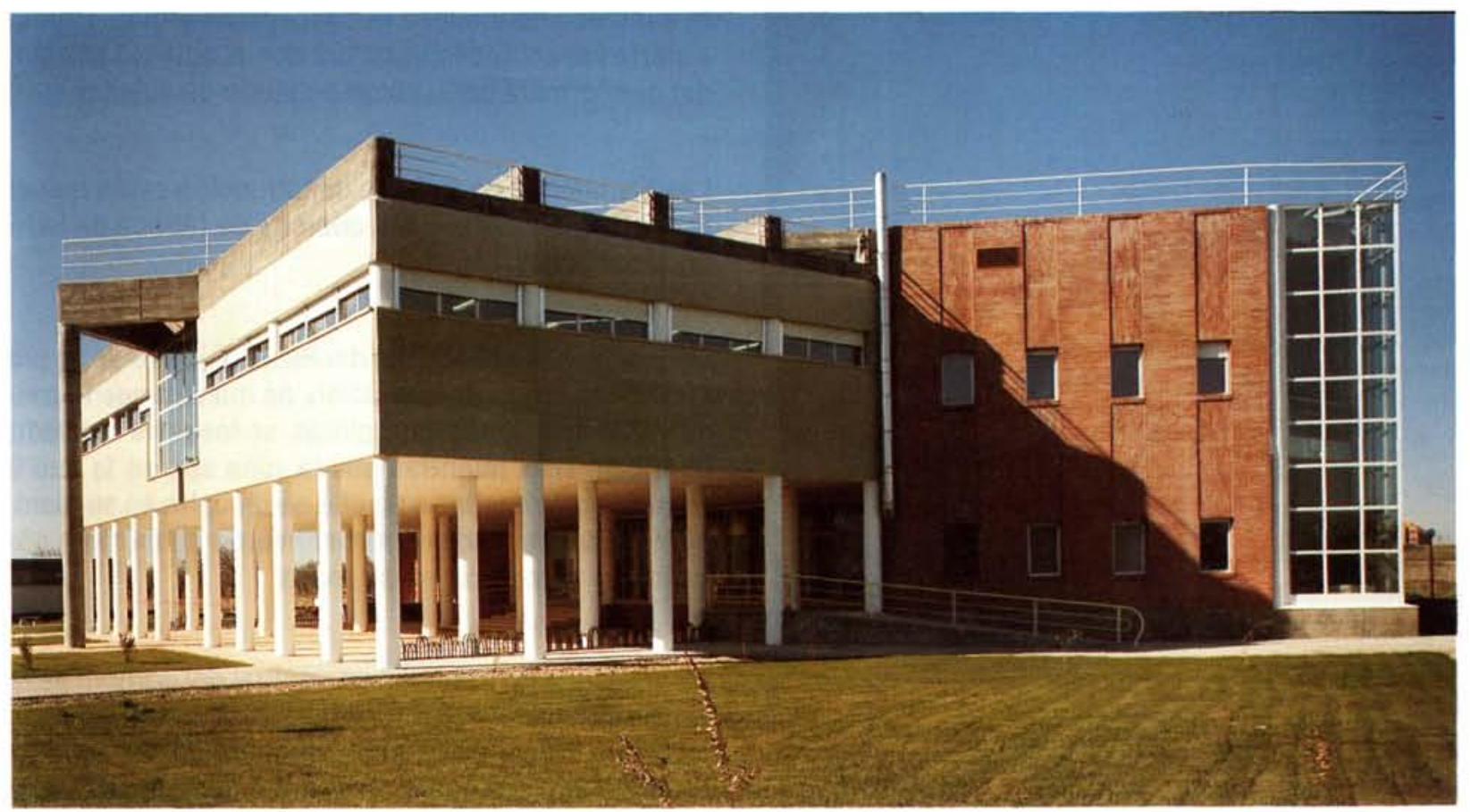




\section{GENERALIDADES}

El Campus de la Universidad de Alcalá de Henares incluye edificios situados en la ciudad de Alcalá, y en una extensa finca de más de 250 hectáreas situada hacia el noroeste de la ciudad, limitada por la variante de la Nacional II y la carretera de Alcalá a Meco.

En el Campus externo están ubicadas las Facultades de Medicina y Farmacia en edificios de reciente construcción, y la Facultad de Ciencias que ocupa un edificio de más de $14.000 \mathrm{~m}^{2}$, proyectado hace años para dependencias militares y adaptado para uso docente.

La nueva organización departamental de la enseñanza y la investigación exigía la remodelación total del edificio de la Facultad de Ciencias. Para acometer esta obra era necesario construir un edificio de nueva planta destinado a pabellón de aulas, y liberar asi del Programa de la Facultad estas dependencias.

En el año 1987 se determinó el Programa, y se desarrolló el Proyecto por la Unidad Técnica Universitaria. Las obras se adjudicaron en octubre del 87 , con un plazo de ejecución de doce meses.

La obra ha entrado en funcionamiento en noviembre del 88 , y el presupuesto total, incluyendo obras de urbanización y ajardinamiento de las zonas de influencia, ha sido de 163 millones de pesetas.

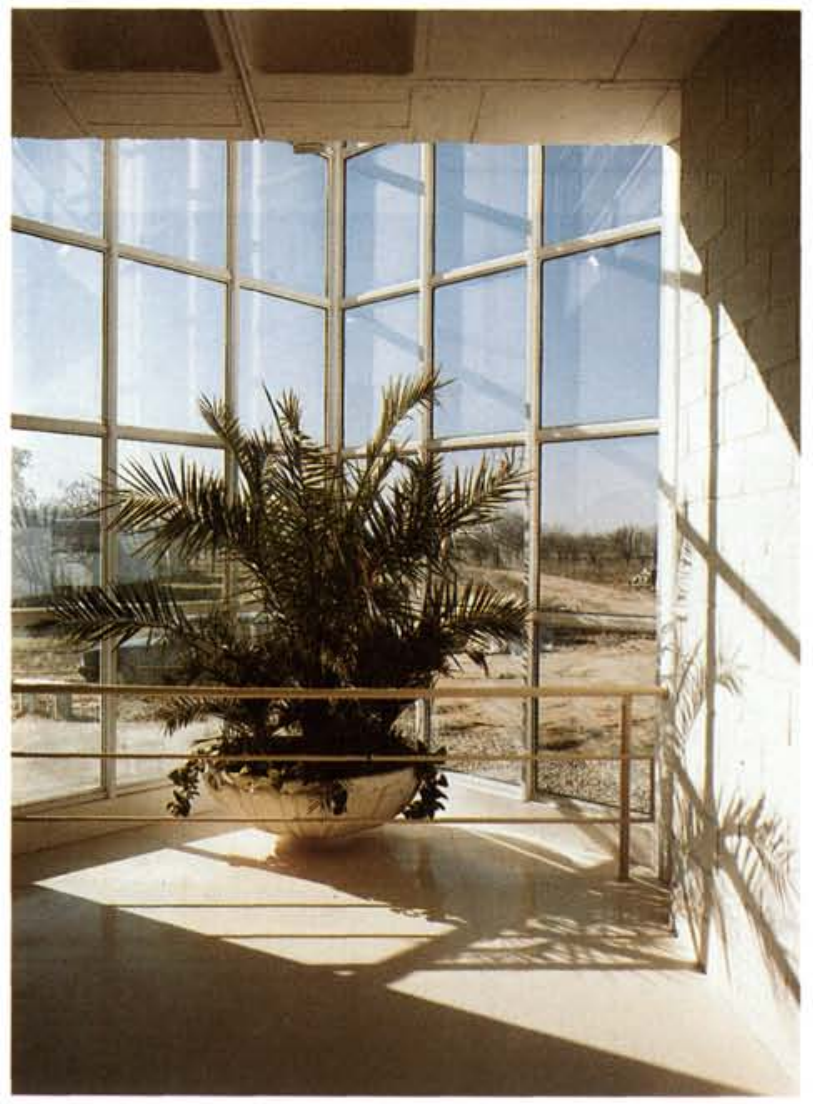

(C) Consejo Superior de Investigaciones Científicas Licencia Creative Commons 3.0 España (by-nc)

\section{PROGRAMA Y ORGANIZACIÓN}

El programa de necesidades incluye aulas de muy diferente capacidad, y por limitaciones presupuestarias en la planta baja se ha organizado un porche con posibilidad de fácil conversión en nuevas aulas, que amplien la capacidad del edificio.

La capacidad total es de 1.000 alumnos, ampliable hasta 1.500 alumnos por turno.

Como enfoque fundamental del Proyecto nos propusimos que el diseño consiguiese óptimas soluciones funcionales con la máxima diafanidad y luminosidad en las zonas de circulación.

La organización es en dos plantas. Los servicios sanitarios se disponen sólo en la planta baja, por resultar más funcionales, y se sitúan las aulas de mayor capacidad en planta primera.

La decisión exige mayor amplitud de las escaleras para una adecuada evacuación, necesitando amplias zonas de circulación en las dos plantas.

Estas exigencias funcionales justifican la pretendida diafanidad espacial.

\section{COMPOSICIÓN DEL PROYECTO}

El emplazamiento del edificio aconseja tener en cuenta e incluso condiciona la concepción arquitectónica a cierta capacidad integradora con el edificio antiguo, del que formará parte como pabellón de aulas (Fig. 1).

Las Facultades de reciente construcción están resueltas con claros volúmenes cúbicos de fábrica de ladrillo a cara vista.

La concepción del edificio del Pabellón de aulas se proyecta como nexo de transición, de manera que la zona norte, de dos plantas completas, se resuelve con ladriIlo a cara vista mientras que la zona sur, en la que la planta baja se destina a porche, se resuelve en su planta primera con fachada de revestimiento de mortero con resinas y coloreado en su masa.

La necesaria continuidad proyectual del edificio se resuelve con un pilar exento centrado en la fachada principal que completa el cuadrado perfecto de la planta. La cubierta plana completa el cubo geométrico conte- 


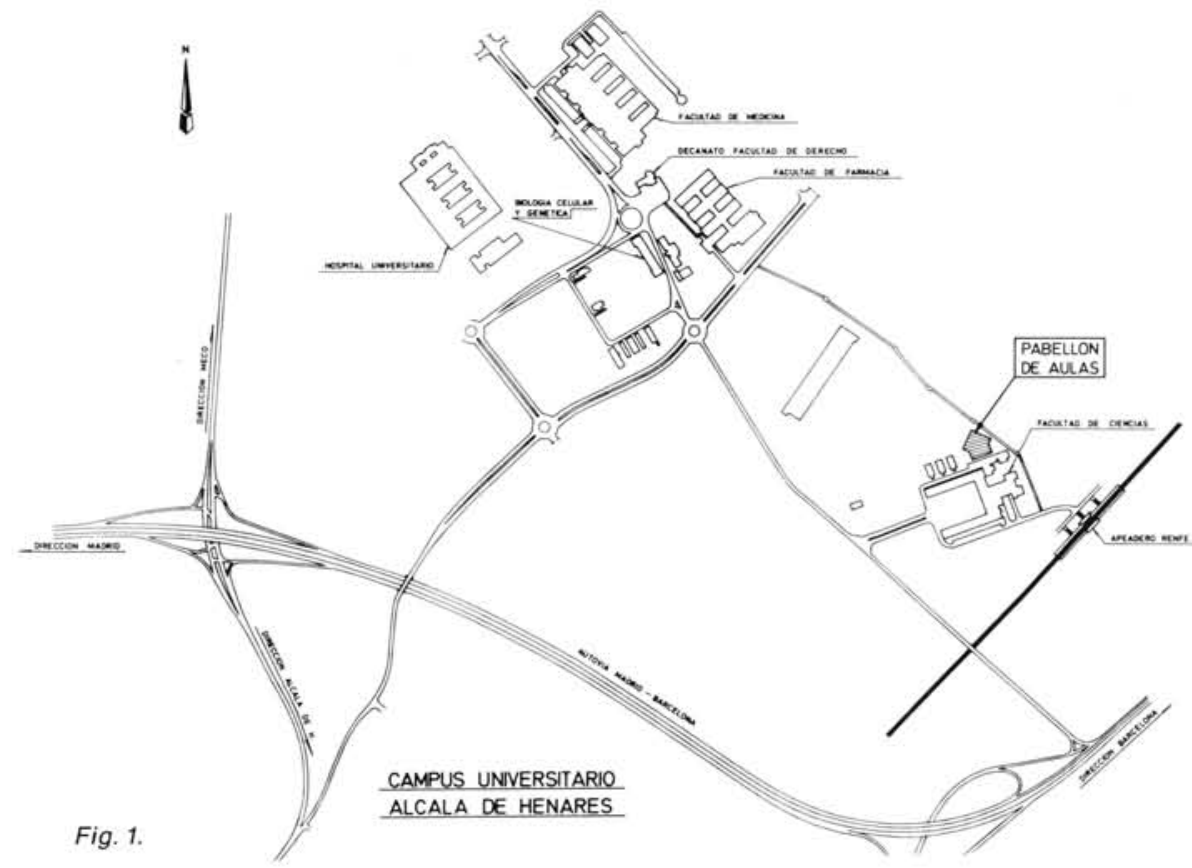

nedor, que se remata con una barandilla de protección que remarca el giro de $45^{\circ}$ de los ejes de simetria conceptual.

Las aulas de gran capacidad de la planta primera, en la zona sur, se destacan resaltando las vigas de hormigón armado en cubierta. Esta solución permite disponer una penetración de luz alta para conseguir una mejor uniformidad de la iluminación natural. El forjado del techo de las aulas se dispone a diferente cota siguiendo la diagonal del aula, que es la alineación de fachada del cuadrado girado 45 grados respecto a la fachada principal del edificio.

\section{SOLUCIONES VOLUMÉTRICAS DE LAS AULAS}

Partiendo de conseguir una composición arquitectónicamente creativa ha sido una preocupación obsesiva proyectual la funcionalidad ambiental, de manera que las aulas reúnan buenas condiciones de iluminación natural-artificial, óptimas condiciones acústicas y cuenten con la necesaria renovación de aire individualizada.

Se ha partido de un esquema de planta triangular, achaflanada en la zona del profesor para colocar el encerado. Esta solución consigue unos despachos para el profesorado, en cada unidad de aula.

La inclinación de los muros o tabiques laterales colaboran a direccionar la atención de los alumnos, y evi-

(c) Consejo Superior de Investigaciones Científicas Licencia Creative Commons 3.0 España (by-nc) tan los reflejos de las ventanas en la zona de pizarra y pantalla.

La utilización continua de medios audiovisuales en la exposición de las clases, proyectores de diapositivas y retroproyectores de acetatos, exigen utilizar la iluminación natural tamizada, y complementarla con luz ar-

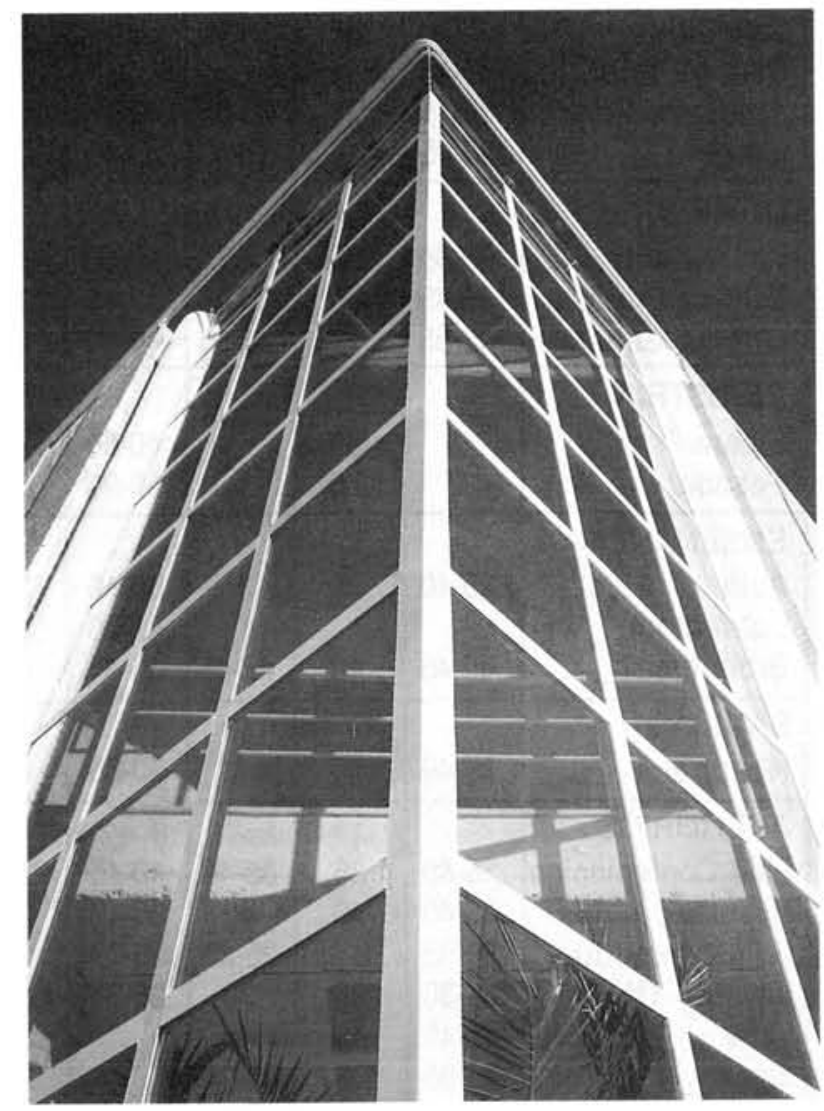

http://informesdelaconstruccion.revistas.csic.es 
tificial. Los huecos están protegidos con micropersianas enrollables, y los capialzados colocados al exterior mejoran el aislamiento térmico en el interior de las aulas.

Se ha estudiado la altura libre de cada planta según la capacidad de las aulas (en la planta baja 3,50 $\mathrm{m}$ y en la primera hasta $4,50 \mathrm{~m}$ ) para conseguir proporciones suficientemente ajustadas al diagrama de Bolt. Partiendo de esta volumetría se ha mejorado el acondicionamiento con materiales de buena capacidad de absorción, con lo que se ha ajustado la curva de frecuenciareverberación a los tiempos óptimos para la inteligibilidad de la palabra, incluso en las aulas de gran capacidad $\sin$ necesidad de utilizar medios auxiliares electro-acústicos (Fig. 2).

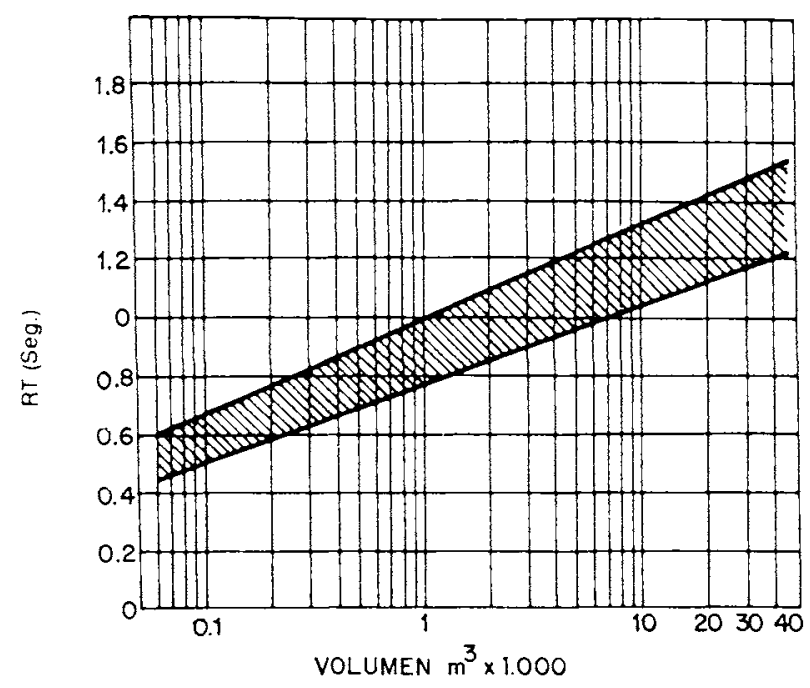

Fig. 2.-Tiempo de reverberación óptimo para palabra.

NIVELES SONOROS RECOMENDADOS PARA VARIOS ESPACIOS INTERIORES

\begin{tabular}{|c|c|c|c|c|c|c|c|c|c|c|c|c|}
\hline & $\begin{array}{c}\text { Knudsen } \\
\text { Harns } \\
1950 \\
d B(A)\end{array}$ & $\begin{array}{c}\text { Beranek } \\
1953 \\
d B(A)\end{array}$ & $\begin{array}{c}\text { Beranek } \\
1957 \\
d B(A)\end{array}$ & $\begin{array}{c}\text { Lawrence } \\
1962 \\
d B(A)\end{array}$ & $\begin{array}{c}\text { Kosten } \\
\text { Van Os } \\
1962 \\
d B(A)\end{array}$ & $\begin{array}{c}\text { Ashrae } \\
1967 \\
d B(A)\end{array}$ & $\begin{array}{c}\text { Denisor } \\
1970 \\
d B(A)\end{array}$ & $\begin{array}{c}\text { Kryter } \\
1970 \\
d B(A)\end{array}$ & $\begin{array}{c}\text { Beranek } \\
1971 \\
d B(A)\end{array}$ & $\begin{array}{c}\text { Doelle } \\
1972 \\
d B(A)\end{array}$ & $\begin{array}{l}\text { Wood } \\
1972 \\
d B(A)\end{array}$ & $\begin{array}{c}\text { Rettinger } \\
1973 \\
d B(A)\end{array}$ \\
\hline $\begin{array}{l}\text { RESIDENCIA } \\
\text { Dormitorio } \\
\text { Sala de estar } \\
\text { Apartamento } \\
\text { Hotel }\end{array}$ & $\begin{array}{l}35-45 \\
35-45 \\
35-45 \\
35-45\end{array}$ & $\begin{array}{l}35 \\
35\end{array}$ & $\begin{array}{l}35-45 \\
35-40 \\
35-40\end{array}$ & $\begin{array}{c}25 \\
40 \\
30 \\
35-40\end{array}$ & $\begin{array}{l}30 \\
35\end{array}$ & $\begin{array}{l}25-35 \\
30-40 \\
35-45 \\
35-45\end{array}$ & & $\begin{array}{l}40 \\
40 \\
48 \\
38\end{array}$ & $\begin{array}{l}34-47 \\
38-47 \\
34-47 \\
34-47\end{array}$ & $35-45$ & $\begin{array}{c}35 \\
40 \\
30-40\end{array}$ & $\begin{array}{c}34-42 \\
38-42 \\
42\end{array}$ \\
\hline $\begin{array}{l}\text { COMERCIO } \\
\text { Restaurante } \\
\text { Oficina Privada } \\
\text { Oficina General }\end{array}$ & $\begin{array}{l}50-55 \\
40-45 \\
45-55\end{array}$ & $\begin{array}{l}55 \\
50\end{array}$ & $\begin{array}{c}55 \\
30-45 \\
40-55\end{array}$ & $\begin{array}{l}40-60 \\
35-45 \\
40-60\end{array}$ & $\begin{array}{c}50 \\
30-45 \\
60\end{array}$ & $\begin{array}{l}40-55 \\
25-35 \\
35-65\end{array}$ & $\begin{array}{l}40-45 \\
50-60\end{array}$ & $\begin{array}{c}55 \\
35 \\
35-40\end{array}$ & $\begin{array}{l}42-52 \\
38-47 \\
42-52\end{array}$ & $\begin{array}{l}45-60 \\
30-45 \\
45-55\end{array}$ & $\begin{array}{l}45-50 \\
40-45 \\
45-55\end{array}$ & $\begin{array}{l}50 \\
46 \\
50\end{array}$ \\
\hline $\begin{array}{l}\text { INDUSTRIA } \\
\text { Ligera } \\
\text { Pesada }\end{array}$ & & $\begin{array}{l}50 \\
75\end{array}$ & & $\begin{array}{l}40-60 \\
60-90\end{array}$ & 70 & & 85 & & $\begin{array}{l}52-61 \\
66-80\end{array}$ & & $\begin{array}{l}55-65 \\
60-75\end{array}$ & 70 \\
\hline $\begin{array}{l}\text { ENSEÑANZA } \\
\text { Aula } \\
\text { Laboratorio } \\
\text { Biblioteca }\end{array}$ & $\begin{array}{l}35-40 \\
40-45\end{array}$ & $\begin{array}{l}35 \\
40\end{array}$ & $\begin{array}{c}35 \\
42-45\end{array}$ & $\begin{array}{l}30-40 \\
40-50 \\
35-45\end{array}$ & $\begin{array}{l}30 \\
35\end{array}$ & $\begin{array}{l}35-45 \\
40-50 \\
35-45\end{array}$ & $40-50$ & $\begin{array}{l}35 \\
40\end{array}$ & $\begin{array}{l}38-47 \\
47-46 \\
38-47\end{array}$ & $\begin{array}{c}35 \\
40-45\end{array}$ & $\begin{array}{l}35-45 \\
45-50 \\
40-45\end{array}$ & $\begin{array}{l}38 \\
42 \\
42\end{array}$ \\
\hline $\begin{array}{l}\text { SANIDAD } \\
\text { Hospital }\end{array}$ & $35-40$ & 40 & 42 & $20-35$ & 35 & $30-45$ & & 40 & $34-47$ & 40 & $40-45$ & 38 \\
\hline $\begin{array}{l}\text { AUDITORIOS } \\
\text { Sala Conferencias } \\
\text { Iglesia } \\
\text { Sala conciertos } \\
\text { Estudio TV } \\
\text { Cine } \\
\text { Teatro }\end{array}$ & $\begin{array}{l}35-40 \\
35-40 \\
30-35 \\
25-30 \\
35-40 \\
30-35\end{array}$ & $\begin{array}{c}35 \\
40 \\
30-35 \\
30 \\
40 \\
35\end{array}$ & $\begin{array}{c}35-40 \\
40 \\
25-35 \\
30 \\
40 \\
30-35\end{array}$ & $\begin{array}{l}40-45 \\
35-40 \\
25-35 \\
25-35\end{array}$ & $\begin{array}{l}35 \\
30 \\
30 \\
35 \\
25\end{array}$ & $\begin{array}{l}30-40 \\
25-35 \\
25-35 \\
35-45 \\
35-45 \\
30-40\end{array}$ & & $\begin{array}{c}38 \\
40 \\
28-35 \\
28 \\
40 \\
33\end{array}$ & $\begin{array}{l}30-42 \\
30-42 \\
21-30 \\
21-34 \\
\\
30-34\end{array}$ & $\begin{array}{c}35-45 \\
35-40 \\
25-35 \\
30-35 \\
40 \\
30-35\end{array}$ & $\begin{array}{c}35-45 \\
35-40 \\
30-35 \\
35 \\
35-40\end{array}$ & $\begin{array}{c}38-42 \\
34 \\
34-38 \\
38 \\
34\end{array}$ \\
\hline
\end{tabular}




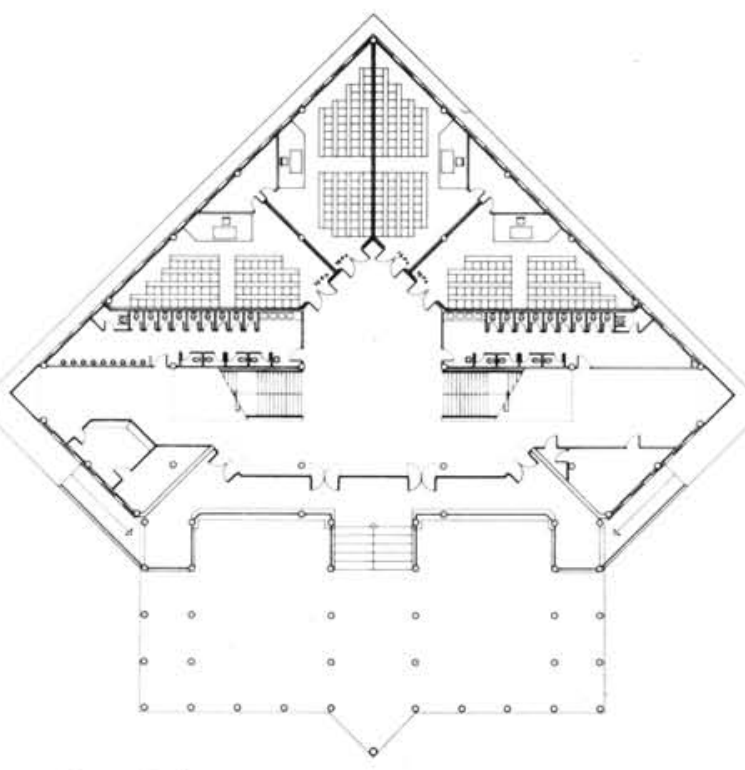

Planta baja
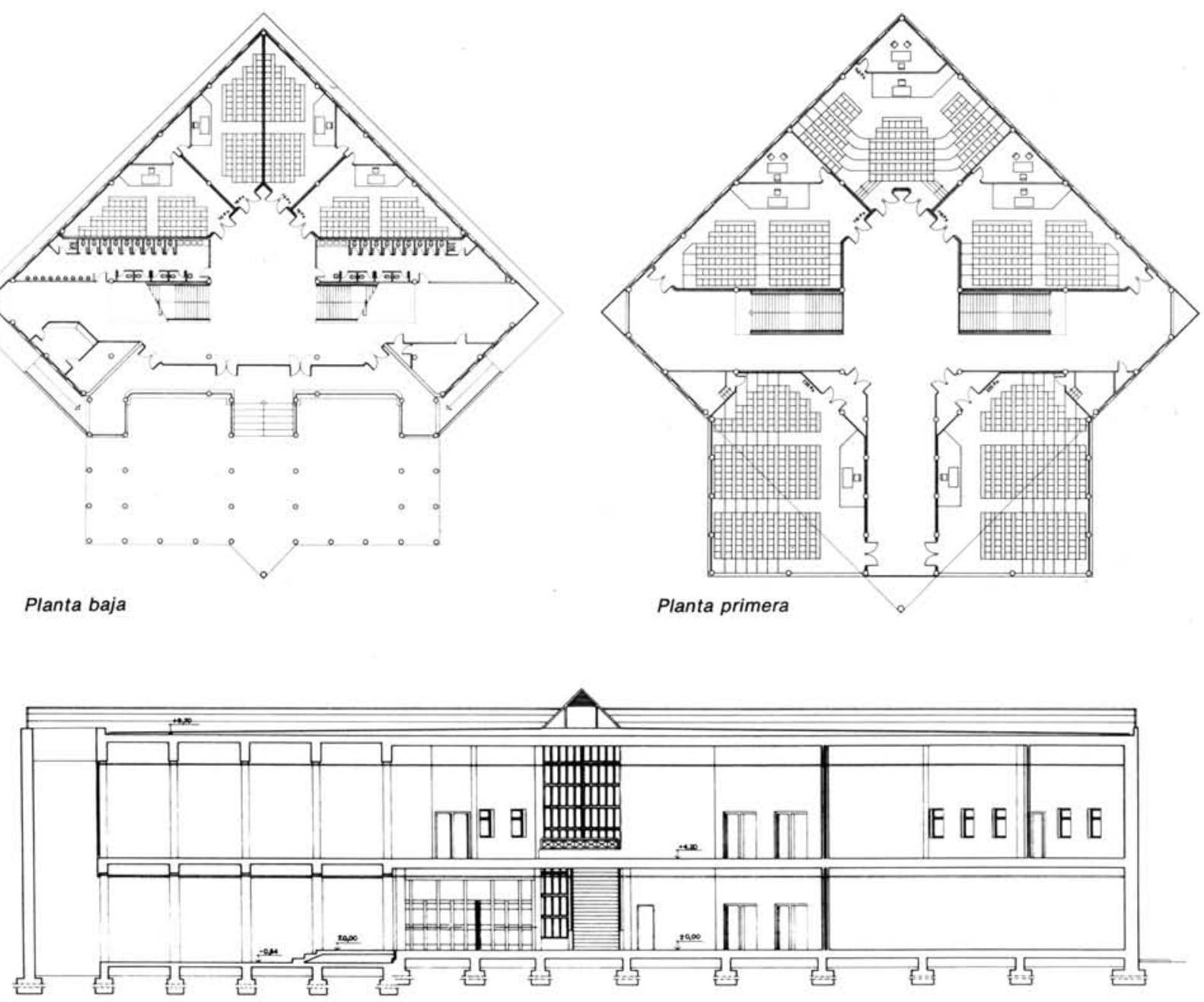

Sección $C \cdot C$

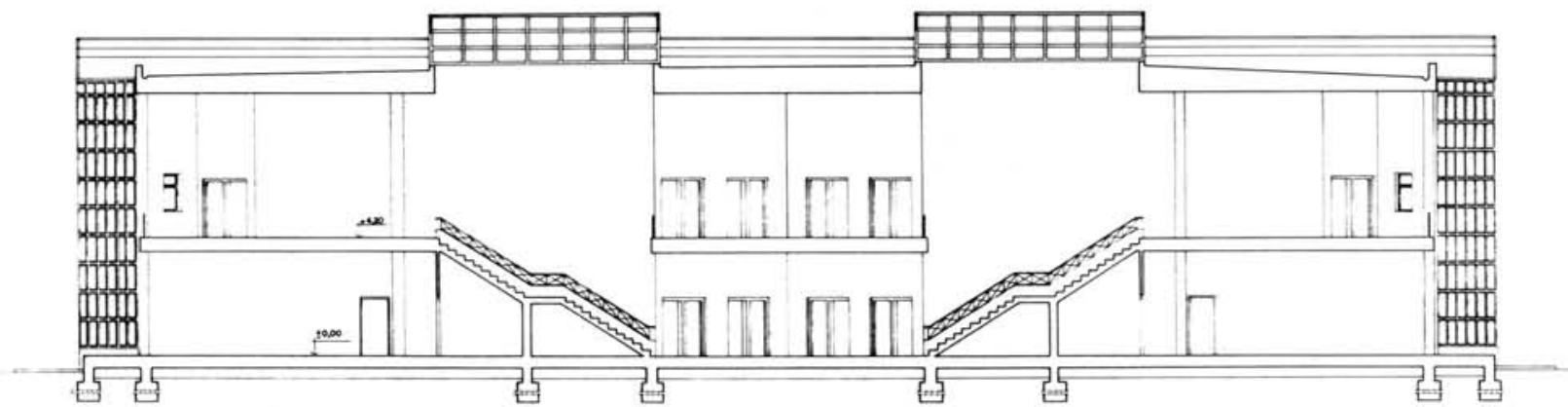

Sección $B \cdot B$

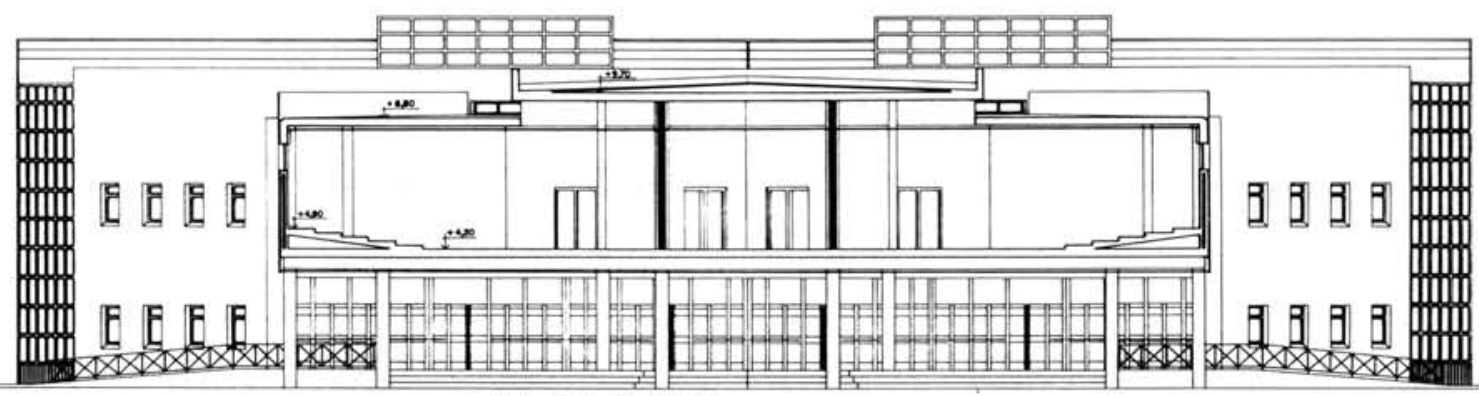

Sección A - A

PABELLON DE AULAS

FACULTAD DE CENCLAS

UNMERSDAD ALCALA DE HENARES

(c) Consejo Superior de Investigaciones Científicas Licencia Creative Commons 3.0 España (by-nc)
SECCIONES

ESCALA $=1: 100$ 

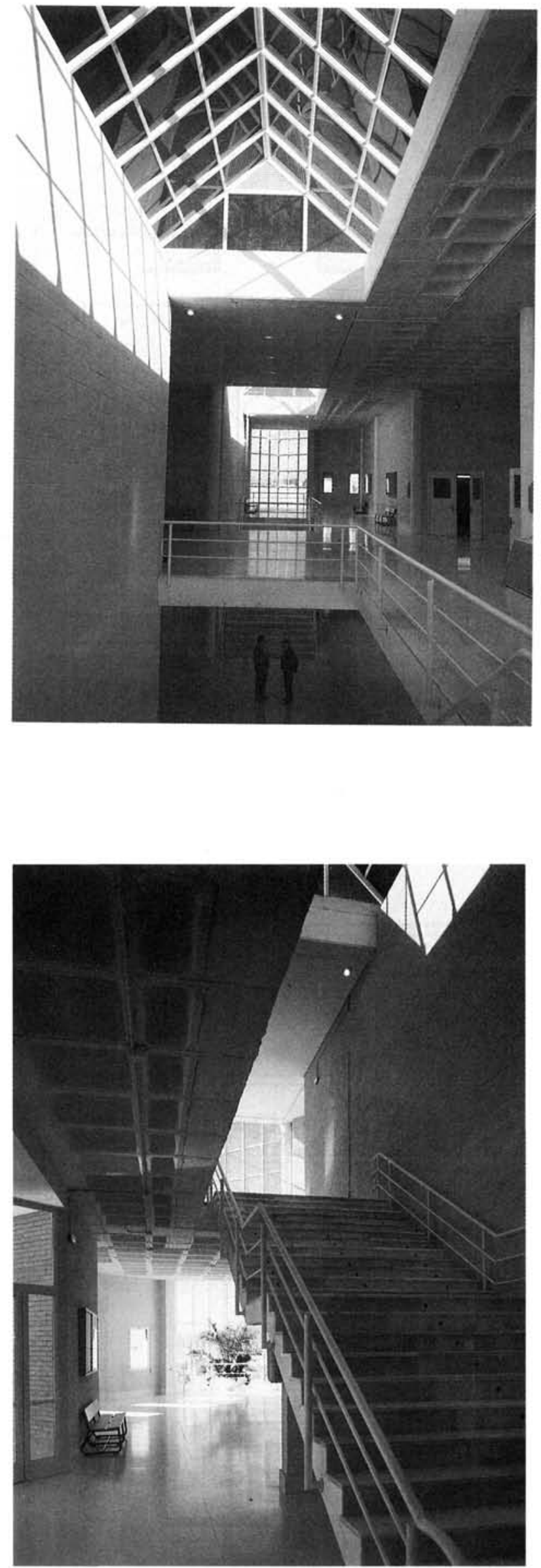

(C) Consejo Superior de Investigaciones Científicas Licencia Creative Commons 3.0 España (by-nc)

\section{CONDICIONES AMBIENTALES}

Sobre las líneas básicas del diseño que se han mencionado se ha pretendido analizar el acondicionamiento ambiental en sus distintas facetas, de forma que cada aula de gran capacidad, o cada dos de las menores, se consideren una unidad sectorizada:

- En cuanto a la Norma NBE-CPI.82, tienen doble acceso, con puertas de apertura hacia el exterior de tablero macizo y material intumescente, RF-30.

- Cuentan con sistema de aireación forzada, que de acuerdo a la norma IT.IC.02 consigue una ventilación de $5 \mathrm{dm}^{3} / \mathrm{s}$ por persona.

- La iluminación natural-artificial se complementa para conseguir tamizar la luz natural y utilizar proyectores y retroproyectores.

- Cada unidad tiene un local propio para almacén de medios, material docente y despacho de profesortutoria.

- El acondicionamiento acústico permite una audición inteligible, sin necesidad de utilizar medios electroacústicos.

La iluminación natural se tensiona diferenciando las zonas comunes y las aulas.

Se pretende la integración en el paisaje en las circulaciones por medio de grandes ventanales: frontal en el eje principal, y en diedro de doble altura en el eje transversal; en cubierta con los lucernarios de metacrilato tintado en verde sobre las escaleras. La inclinación de $45^{\circ}$ de las planchas de metacrilato, de pieza única en vertical, y una permanente aireación garantizan que no se desprendan gotas en el interior por humedad de condensación.

En las aulas la iluminación natural se ha reducido a la suficiente, para evitar sensación de claustrofobia.

La iluminación artificial también está claramente tensionada ya que en las aulas se consiguen hasta 800 lux con luminarias fluorescentes, $y$ en las zonas comunes sólo hay puntos incandescentes ocultos, direccionados en las escaleras y muy concentrados, de halógenos, en los accesos.

http://informesdelaconstruccion.revistas.csic.es 


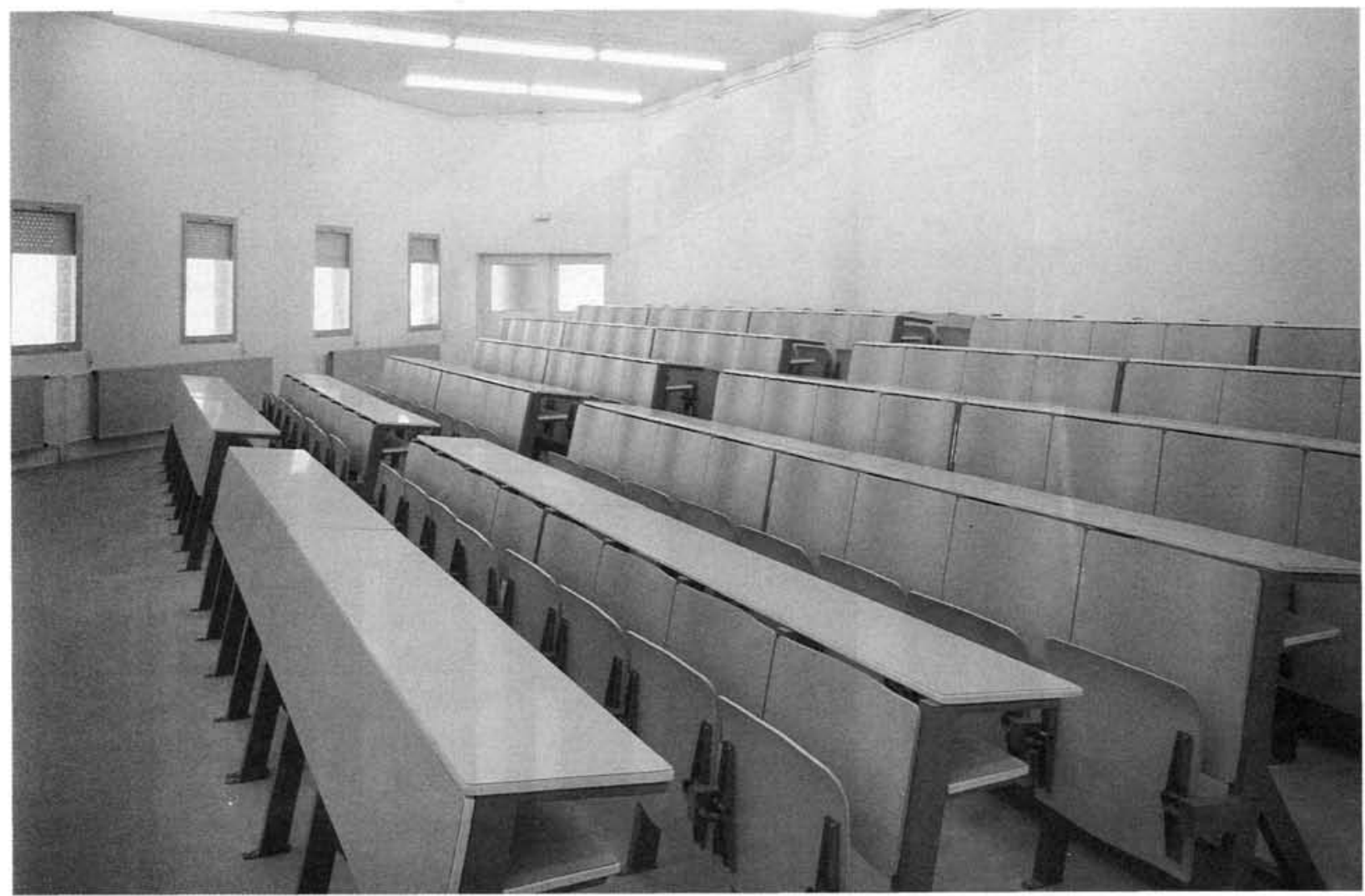

\section{ACONDICIONAMIENTO ACÚSTICO}

Para conseguir buenas condiciones acústicas en un local es necesario partir del diseño.

\subsection{La forma de las aulas}

Todas las superficies planas son rectas y las zonas próximas al profesor son oblicuas a la dirección del sonido directo.

La proporción en planta, de ancho superior al fondo, mejora el ambiente coloquial, además de conseguir mejor visibilidad direccional.

La altura libre ajusta suficientemente las proporciones con el largo y el ancho de cada aula al diagrama de Bolt, de forma compatible con las exigencias de iluminación. Se escalonan para aumentar la visibilidad y por tanto mejorar la audición. Las filas posteriores pierden menos energía sonora del sonido directo.

No se producen "ecos" - percepción de sonidos con diferencias mayores de 50 milisegundos-, lo que corresponde a diferencia de caminos entre los sonidos directo y reflejado superiores a $17 \mathrm{~m}$.

(c) Consejo Superior de Investigaciones Científicas Licencia Creative Commons 3.0 España (by-nc)

\subsection{Nivel de ruido de fondo, en las aulas}

El nivel de ruido de fondo es silencioso - de media a muerta según el gráfico-. Las aulas de mayor capacidad cuentan con vestibulo de insonorización de la zona más ruidosa (Fig. 3 ).

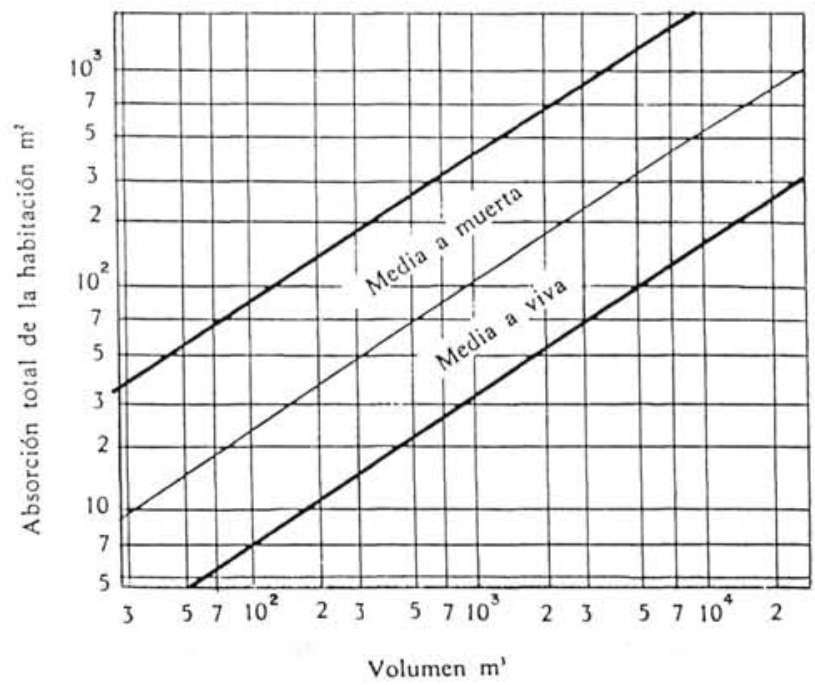

Fig. 3.-Condiciones de habitación: De media a muerta.-Aulas, pasillos, oficinas generales, espacios de ocupación mediana a fuerte. De media a viva.-Salas de conferencias, espacios de tamaño medio a pequeño donde la comunicación verbal es actividad predominante. 

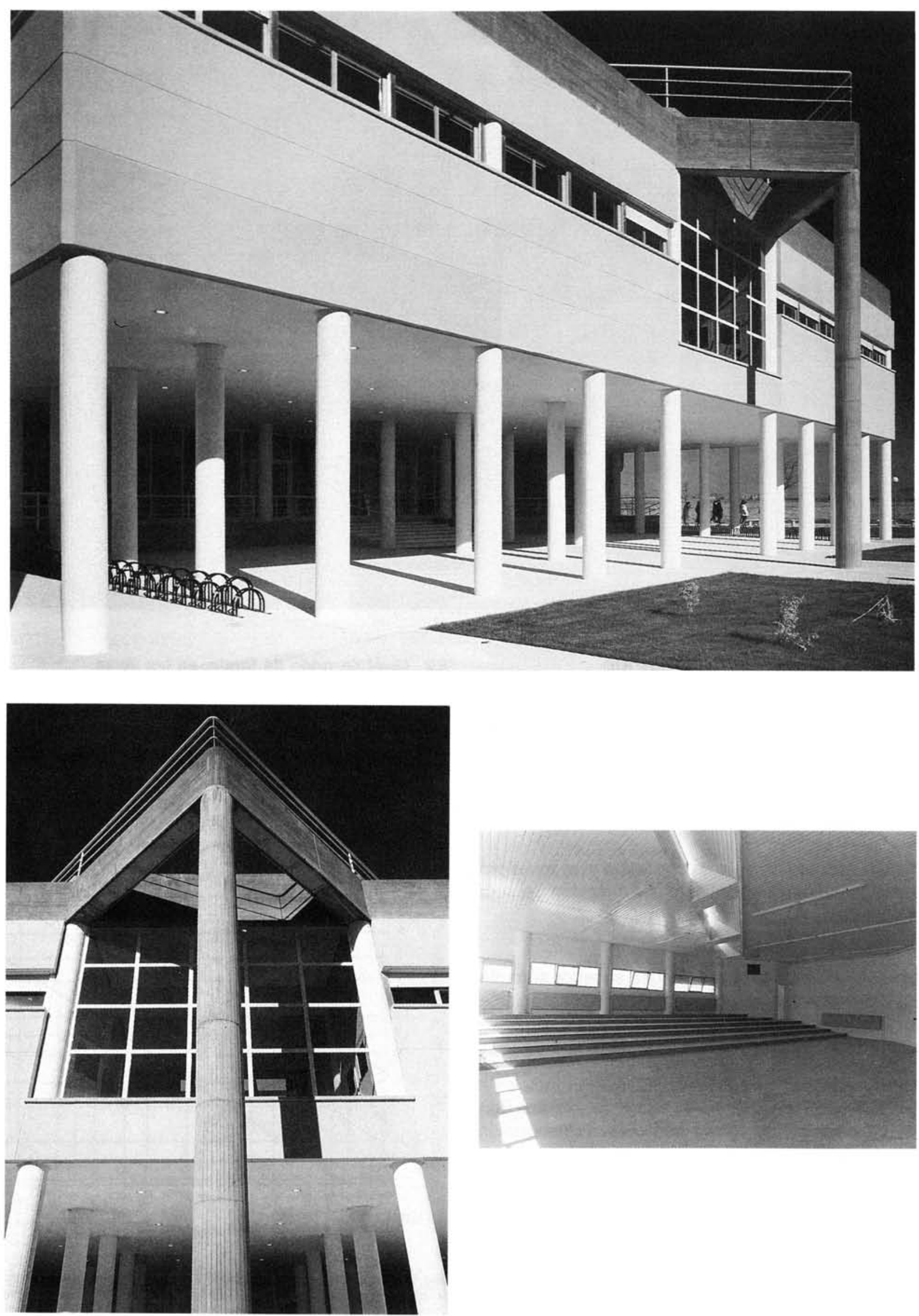
Las soluciones constructivas consiguen menor nivel de inmisión ( $\mathrm{L}_{\text {eq }}$ ) que los $40 \mathrm{dBA}$, recomendados por la Norma NBE-CA-88.

El aislamiento a ruido aéreo de los muros de fachada (R) supera los $50 \mathrm{dBA}$, por lo que cumple holgadamente con los 30 mínimos de la Norma.

La tabiqueria de distribución se ha estudiado con cuidado. Está formada por varias capas, de diferente espesor y material, tabicón de ladrillo cerámico en el interior, cámara rellena con panel absorbente y bloque de hormigón a las zonas comunes.

Esta solución mejora el aislamiento en relación con una pared simple del mismo espesor total. A falta de ensayo de laboratorio, considerando las fórmulas logaritmicas de la NBE-CA-88, y valores comparativos de publicaciones especializadas el valor de $\mathrm{R}$ estará cercano a $50 \mathrm{dBA}$.

Los huecos exteriores están cerrados con ventanas de aluminio, de una sola hoja, abatible sobre eje inferior y los capialzados tienen su apertura por el exterior. EI vidrio es "climalit 4, 6, 4" tintado de verde.
Esta carpinteria puede considerarse de la clase A-3, E-2, V-3 para los ensayos de permeabilidad al aire, estanquidad al agua y resistencia al viento.

Las puertas son pesadas, macizas con acabado de melamina, cierre con sellado de junta elástica y puede considerarse con umbral, al existir cubrejuntas entre el pavimento interior blando y el exterior duro.

El pavimento de las aulas es de PVC, "saipolán" en baldosas soldadas, lo que mejora el aislamiento a ruido de impacto. Se coloca falso techo, de material altamente absorbente, "Ondexlam 100".

Considerando todo lo anterior, y suponiendo que en el exterior o en las zonas comunes pueda existir un nivel de ruido superior a $80 \mathrm{dBA}$, se garantiza que el nivel de inmisión ( $L_{e q}$ ) en las aulas será inferior a los 40 dBA, recomendados en la NBE-CA-88.

\subsection{Acondicionamiento fónico de las aulas}

Las aulas serán acústicamente correctas si se consigue una buena distribución del sonido en su interior, de manera que son esenciales:

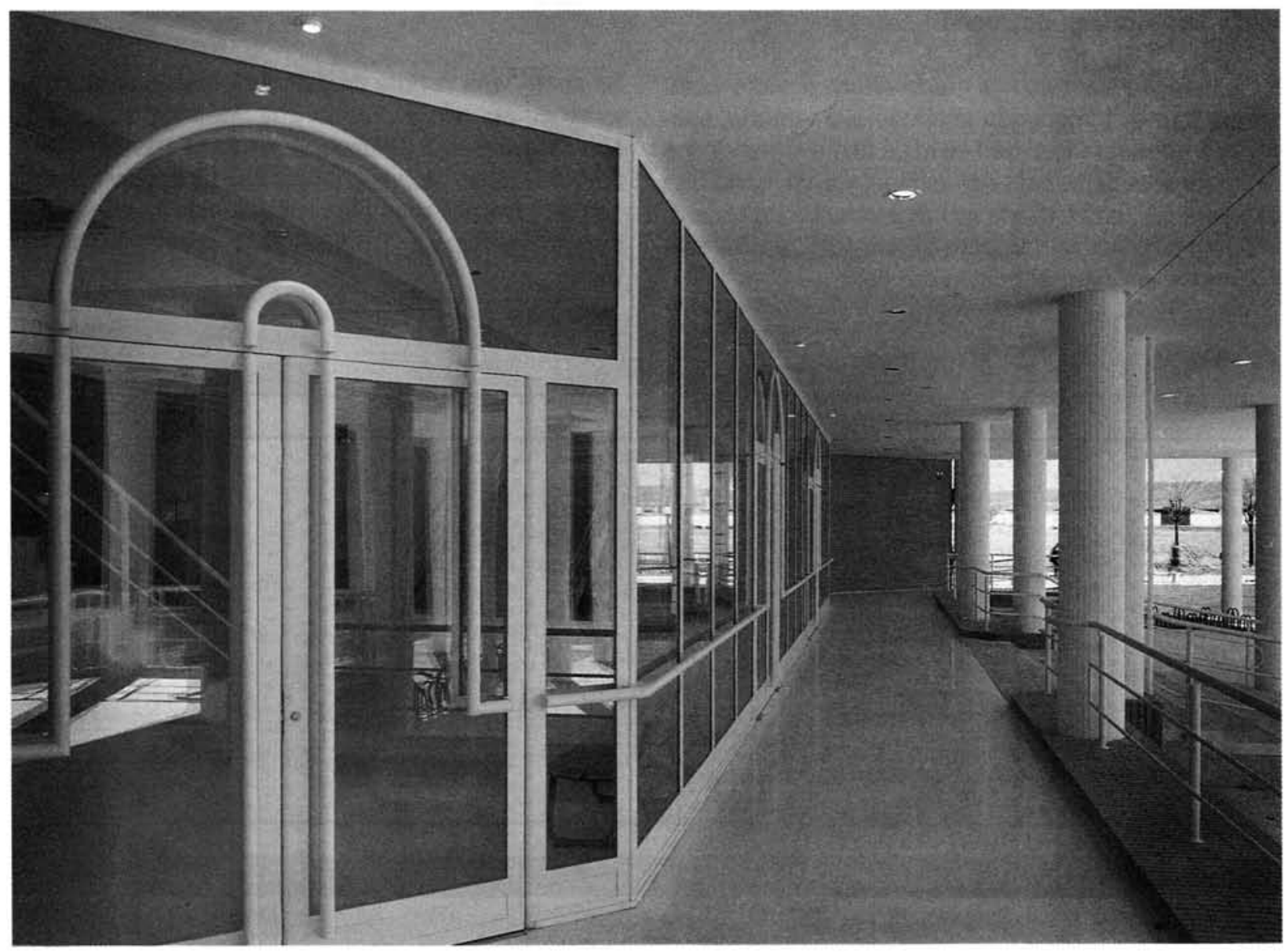


- La intensidad del sonido emitido.

- La inteligibilidad de la palabra.

- El tiempo de reverberación adecuado.

Todas estas cuestiones, entre otras, tienen mucho que ver con la volumetría de las aulas, y en consecuencia es necesario analizar cada una, según su volumen, su capacidad y los materiales de revestimiento.

Como ejemplo vamos a referir el caso de una de las aulas grandes de la planta primera.

Partimos de una serie de condicionantes de diseño que determinan los parámetros de dimensiones, altura, y la distribución de los asientos.

Decidido para el pavimento un material blando, la corrección acústica necesaria para ajustar la curva real de reverberación-frecuencia a la óptima, se realiza con un material de recubrimiento de falso techo, por razones fundamentales de conservación y mantenimiento. Los paramentos verticales en consecuencia se tratan con pinturas, de forma que el frente del profesor resulte reflectante, y suficientemente absorbentes los laterales que se utilizan de percheros. El paramento de fondo tiene poca incidencia, debido al escalonamiento del aula y la absorción del techo.

El falso techo se sitúa a la mayor altura posible, compatible con la iluminación alta, complementaria, conseguida entre las vigas de $1,20 \mathrm{~m}$ de canto situando los forjados en la parte baja en fachada, y en la parte alta en el interior. Esta entrada de luz, cerrada con metacrilato coloreado en verde, como el "climalit", uniformiza la iluminación natural dentro del aula.
Se ha elegido un material, "ondexlam 100" de lamas de PVC, perforado y trasdosado con panel de fibra de vidrio de $20 \mathrm{~mm}$ de espesor.

Este material es desmontable fácilmente reemplazable, y dispone de una gama de colores amplia, y está clasificado como M-1 autoextinguible, lo que resulta fundamental para el comportamiento ante el fuego (Fig. 5).

Se acompañan los gráficos siguientes:

- Tiempo óptimo de reverberación para locales en los que es fundamental la inteligibilidad de la palabra. Para el volumen del aula considerada, el tiempo óptimo de reverberación debe estar comprendido entre 0,7 y $1 \mathrm{~s}$.

- Superficie de unidades de absorción, total del aula. Para el volumen del aula, considerando un nivel de ruido de fondo adecuado, condiciones de la habitación de media a muerta, la superficie total de unidades de absorción debe estar comprendida entre 100 y un máximo de $400 \mathrm{~m}^{2}$.

- Se emplea la fórmula aproximada de $T=0,16$ VIA de Sabine.

Se acompaña la hoja de cálculo donde puede observarse como, considerando una ocupación media del aula, la superficie de absorción con el material decidido para el falso techo consigue ajustar la curva de tiempos de reverberación para las distintas frecuencias al tiempo óptimo, y a la superficie total de absorción óptima.

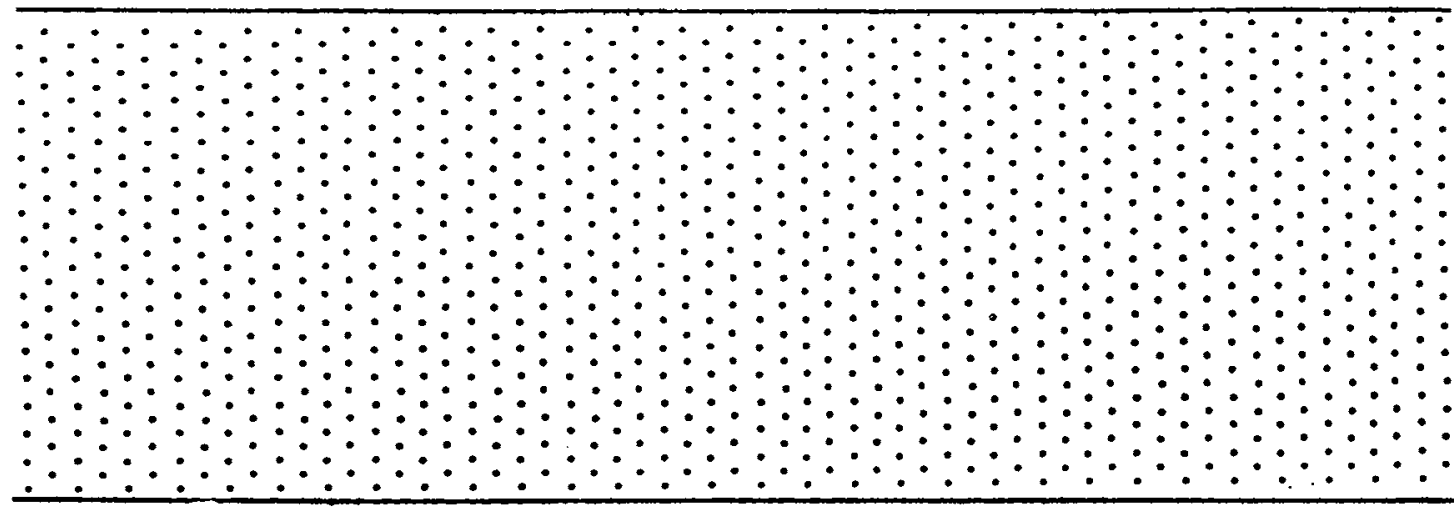

LAMA DE PVC - ONDEXLAM 100

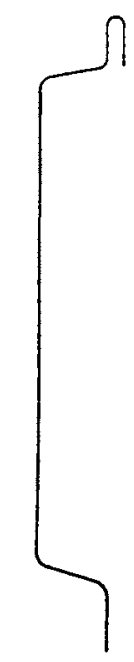

CORRECCION ACUSTICA = ONDEXLAM + FIBRA DE VIDRIO Material M-1 
HOJA DE CALCULO

\begin{tabular}{|c|c|c|c|c|c|c|c|c|c|c|c|c|c|}
\hline \multirow{2}{*}{ MATERIALES } & \multirow{2}{*}{$\begin{array}{l}\text { SUP } \\
\mathrm{m}^{2}\end{array}$} & \multicolumn{2}{|c|}{125} & \multicolumn{2}{|c|}{250} & \multicolumn{2}{|c|}{500} & \multicolumn{2}{|c|}{1.000} & \multicolumn{2}{|c|}{2.000} & \multicolumn{2}{|c|}{4.000} \\
\hline & & $\alpha$ & $\alpha \mathbf{S}$ & $\alpha$ & $\alpha \mathbf{S}$ & $\alpha$ & $\alpha \mathbf{S}$ & $\alpha$ & $\alpha \mathbf{S}$ & $\alpha$ & $\alpha \mathbf{S}$ & $\alpha$ & $\alpha \mathbf{S}$ \\
\hline PAVIMENTO (de PVC) & 200 & 0,01 & 2 & 0,01 & 2 & 0,01 & 2 & 0,02 & 4 & 0,02 & 4 & 0,01 & 2 \\
\hline PAREDES (pintura) & 130 & 0,01 & 1,3 & 0,01 & 1,3 & 0,02 & 2,6 & 0,03 & 3,9 & 0,04 & 5,2 & 0,05 & 6,5 \\
\hline VENTANAS (aluminio) & 25 & 0,04 & 1 & 0,04 & 1 & 0,03 & 0,75 & 0,03 & 0,75 & 0,02 & 0,5 & 0,02 & 0,5 \\
\hline PUERTAS (madera) & 10 & 0,09 & 0,9 & 0,09 & 0,9 & 0,08 & 0,8 & 0,09 & 0,9 & 0,10 & 1 & 0,07 & 0,7 \\
\hline $\begin{array}{l}\text { (OCUPACION } \\
\text { MEDIA) }\end{array}$ & $\begin{array}{c}100 \\
\text { al }\end{array}$ & 0,15 & 15 & 0,25 & 25 & 0,35 & 35 & 0,38 & 38 & 0,38 & 38 & 0,35 & 35 \\
\hline (ONDEXLAN) & 200 & 0,86 & 172 & 0,79 & 158 & 0,87 & 174 & 0,99 & 198 & 0,86 & 172 & 0,86 & 172 \\
\hline $\begin{array}{l}\text { UNIDADES } \\
\text { ABSORCION }\end{array}$ & $A_{1}$ & & 20 & & 30 & & 41 & & 48 & & 49 & & 45 \\
\hline $\mathrm{Tr}_{1}=\frac{0,16 \mathrm{~V}}{\mathrm{~A}_{1}}=\frac{144}{\mathrm{~A}_{1}}$ & & & 7,2 & & 4,8 & & 3,5 & & 3 & & 2,9 & & 3,2 \\
\hline 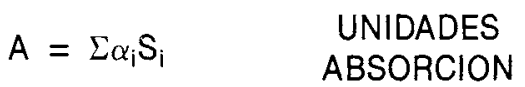 & $A_{2}$ & & 209 & & 204 & & 232 & & 266 & & 238 & & 234 \\
\hline $\mathrm{Tr}_{2}=\frac{0,16 \mathrm{~V}}{\mathrm{~A}_{2}}=\frac{144}{\mathrm{~A}_{2}}$ & & & 0,84 & & 0,91 & & 0,83 & & 0,73 & & 0,84 & & 0,84 \\
\hline $\begin{array}{l}\text { UNIDADES DE } \\
\text { ABSORCION (OPTIMO) }\end{array}$ & & & & & & & $y<$ & & & & & & \\
\hline $\operatorname{Tr}$ (OPTIMO) & & & & & & & $7 y$ & & & & & & \\
\hline
\end{tabular}

Cálculo de acondicionamiento fónico del aula de planta primera, volumen $900 \mathrm{~m}^{3}$ y capacidad 220 puestos escolares.

\section{FICHA TECNICA}

Propiedad:

Equipo técnico:

Autor y director:

Aparejador:

Empresa constructora:

Asesor, estudio acústico:

Control de calidad:

Fecha del proyecto:

Entrada en funcionamiento: Noviembre 1988.
Ministerio de Educación y Ciencia.

Unidad Técnica de la Universidad.

Carmelo Oñate Gómez.

Rodolfo Peña Groth.

Agromán, S. A.

Fernando Calderón.

Cotas Internacional.

Julio 1987. 